\title{
Fracture patterns and bone mass in South African adolescent- mother pairs: the Birth to Twenty cohort
}

\author{
K. Thandrayen $\cdot$ S. A. Norris $\cdot$ L. K. Micklesfield • \\ J. M. Pettifor
}

Received: 13 March 2013 / Accepted: 26 July 2013 / Published online: 14 August 2013

(C) The Author(s) 2013. This article is published with open access at Springerlink.com

\begin{abstract}
Summary The associations of fracture prevalence and bone mass in adolescents with maternal fracture history and bone mass have not been investigated previously in South Africa. Maternal bone mass has a significant inverse association with their adolescents' fracture rates and bone mass across all ethnic groups.

Introduction Differences in fracture rates and bone mass between families and individuals of different ethnic origins may be due to differing lifestyles and/or genetic backgrounds. This study aimed to assess associations of fracture prevalence and bone mass in adolescents with maternal fracture history and bone mass, and sibling fracture history.

Methods Data from 1,389 adolescent-biological mother pairs from the Birth to Twenty longitudinal study were obtained. Questionnaires were completed on adolescent fractures until $17 / 18$ years of age and on sibling fractures. Biological mothers completed questionnaires on their own fractures prior to the age of 18 years. Anthropometric and bone mass data on adolescent-biological mother pairs were collected.

Results An adolescent's risk of lifetime fracture decreased with increasing maternal lumbar spine (LS) bone mineral
\end{abstract}

K. Thandrayen · S. A. Norris $\cdot$ L. K. Micklesfield · J. M. Pettifor MRC/Wits Developmental Pathways for Health Research Unit, Department of Paediatrics, Faculty of Health Sciences, University of the Witwatersrand, Johannesburg, South Africa

S. A. Norris

e-mail: Shane.norris@wits.ac.za

L. K. Micklesfield

e-mail: Lisa.Micklesfield@uct.ac.za

J. M. Pettifor

e-mail: John.pettifor@wits.ac.za

K. Thandrayen $(\bowtie)$

Department of Paediatrics, Chris Hani Baragwanath Hospital, PO

Bertsham, Johannesburg 2013, South Africa

e-mail: Kebashni.thandrayen@wits.ac.za content (BMC; $24 \%$ reduction in fracture risk for every unit increase in maternal LS BMC Z-score) and increased if they were white, male, or had a sibling with a history of fracture. Adolescent height, weight, male gender, maternal bone area and $\mathrm{BMC}$, and white ethnicity were positive predictors of adolescent bone mass. White adolescents and their mothers had a higher fracture prevalence (adolescents $42 \%$, mothers $31 \%$ ) compared to the black (adolescents $20 \%$, mothers $6 \%$ ) and mixed ancestry (adolescents $20 \%$, mothers $16 \%$ ) groups.

Conclusion Maternal bone mass has a significant inverse association with their adolescent off-springs' fracture risk and bone mass. Furthermore, there is a strong familial component in fracture patterns among South African adolescents and their siblings.

Keywords Adolescents · Biological mothers · Bone mass . Family patterns $\cdot$ Fractures $\cdot$ South Africa

\section{Introduction}

Heritability [1,2] and lifestyle factors [3] of both mother during pregnancy and child influence the accrual of peak bone mass and impact the risk of osteoporosis in later adulthood. Intrauterine programming and environmental influences during early childhood may modify peak bone mass accrual. There is no consistent long-term effect of low birth weight on bone mineral density and hip fracture risk later in life [4] but thinness in childhood may be a risk factor for fracture in later life [5]. A meta-analysis and systematic review showed that higher birth weight is associated with greater bone mineral content (BMC) in adulthood [6] and, in almost all of those studies, this relationship was independent of body size. Cooper et al. [7] concluded that infant growth and physical activity in childhood are important determinants of peak bone mass in women. However, it has also been shown that gains in bone 
mineral accretion during childhood via interventions such as increased physical activity and nutrient supplementation may only be transient, thus promoting the hypothesis that bone mass is ultimately governed by a homeostatic system which tends to return towards a yet-to-be defined set point [8]. Whether this set point is genetically predetermined needs to be further investigated. Our research group has shown that heritability of bone area (BA) and BMC by maternal descent is approximately $30 \%$ in South African pre/early pubertal black and white children, despite ethnic differences in both body and bone size, as well as in lifestyle [9]. The pattern of ethnic differences in bone strength in youth $[10,11]$ is similar to the reported ethnic differences in fracture rates in adults [12-14], suggesting that these differences in fracture rates may track back to differences in bone strength in childhood and adolescence.

Although heritability has been shown to be an important determinant of bone mineral accrual and fracture risk in other countries [15], no information is available on the differences in bone mass and fracture patterns between families of different ethnic backgrounds in South Africa. In this study, we were interested in assessing the associations between bone mass and fracture history of mothers with those of their adolescent children. We hypothesized that as there is a strong association between the bone mass measurements of adolescent-biological mother pairs, maternal bone mass will influence fracture prevalence in their adolescent offspring and that a history of fractures in the mother or other siblings will be associated with an increased risk of fractures in the adolescent.

\section{Methods}

\section{Study population}

Data from 1,389 adolescent-biological mother pairs from the Birth to Twenty (Bt20) longitudinal study of child health and development were used. All eligible neonates $(n=3,273)$ born within a 7-week period (April 23 to June 8, 1990) in the greater Johannesburg metropolitan area in South Africa were recruited at birth into the $\mathrm{Bt} 20$ study. Although the total cohort is demographically similar to long-term resident families living in Soweto, Johannesburg, the cohort under represents white children due to white families generally utilizing private practitioners and facilities which were excluded during initial enrolment. To compensate for this, at the age of 10 years, we recruited a supplementary sample of 120 white children born during the same period as the cohort children in 1990 into the bone health sub-study of the Bt20 cohort. Of the 3,273 children in the cohort initially, contact has been maintained with more than $70 \%$ at the age of 16 years. A cohort profile describing the study sample, research objectives and attrition has been documented by Richter et al. [16]. An adolescent's ethnic classification was defined by the race classification currently used in South Africa for demographic and restitution purposes. The South African government currently classifies race into black (B; ethnic Africans), white (W; Europeans, Jews and Middle Easterners), coloured or mixed ancestry (MA; mixed race) and Indian (South Asian), and only adolescents whose parents were classified as being of the same ethnic group were included. Data from 1,389 adolescentbiological mother pairs were analysed for this study. The ethnic breakdown of the study sample was predominantly B $(1,170(84.2 \%))$, with the remainder of the cohort being made up of W (91 (6.6 \%)) and MA (128 (9.2\%)). Indian adolescents and their mothers were excluded as the number of participants was too few to make meaningful comparisons. Children who had chronic diseases such as rheumatoid arthritis, epilepsy and asthma were excluded from the data analyses, as the use of certain medications and immobility are associated risk factors for low bone mass and may increase the incidence of fractures. All subjects provided assent and their parents/guardian provided written, informed consent. Ethical approval for the study was obtained from the University of the Witwatersrand Committee for Research on Human Subjects.

\section{Fracture questionnaire}

A fracture questionnaire was completed by each adolescent with the assistance of his/her parent or caregiver at 15 and 17/ 18 years of age. The questionnaire at age 15 included information on previous fractures from birth until 15 years of age, including site of fracture with the aid of a skeletal diagram, and the causes and age at fracture. At age 17/18, the fracture questionnaire included information on fractures that had occurred since their previous questionnaire. Mothers/caregivers also completed a questionnaire on fractures occurring since birth in the adolescent's sibling(s). Biological mothers completed questionnaires on their own fractures prior to the age of 18 years. Due to the retrospective nature of the fracture data collection, the fractures could not be verified by radiographs.

Anthropometric measurements and dual-energy X-ray absorptiometer-derived parameters

Anthropometric measurements and bone mass data on the subjects at the age of $17 / 18$ years were used for this study. Biological mothers' anthropometric data and bone mass measurements had been collected over 2 years when the adolescents were approximately 13 years of age. Height was measured to the nearest millimetre using a stadiometer (Holtain, Crosswell, UK). Weight was measured to the last $100 \mathrm{~g}$ using a digital scale (Dismed, Halfway House, South Africa), with participants wearing light clothing and no shoes. Tanner staging of pubertal development was assessed by the adolescents privately using a validated protocol based on Tanner's Sexual 
Maturation Scale [17]. This scale, which had been previously validated for black South Africans [18], consists of drawings and explanations of the five Tanner stages of secondary sexual characteristics (breast development in females and genital development for males), ranging from stage 1 (pre-pubertal) through stage 5 (post-pubertal). Same sex researchers were available to assist the adolescents if necessary.

Total body (TB) and lumbar spine (LS) BA and BMC were measured in both the adolescents and biological mothers using a Hologic QDR 4500A dual-energy X-ray absorptiometer according to standard procedures using the same software version for both the adolescents and biological mothers (software version 11.2, Hologic, MA, USA).

\section{Statistical analyses}

The data were analysed using SAS (version 9.3) package. In the descriptive analysis of the adolescent-biological mother pair characteristics, the baseline data were summarized as means (standard deviations). ANOVA was used to test for differences in age and anthropometric measurements; ANCOVA, adjusting for height and weight, was used to test for differences in bone mass (bone mineral content and bone area) measurements between ethnic groups. Bonferonni correction was used for post hoc comparisons of individual groups. Categorical data were summarized as numbers and percentages. Comparisons were made between those who had and had no fracture(s) using chi-square or Fisher's exact analysis. A $p$ value of $<0.05$ was considered to be statistically significant. Ethnicity was dummy coded in all regression models, with whites as the reference group. The pubertal stages of the adolescents were recorded into early puberty (Tanner 1-3) and late puberty (Tanner 4-5) for use in the regression models. Multiple forward selection and backward elimination stepwise regression analyses examined the independent contributions of various factors to adolescent TB and LS BA and BMC, and all variables left in the model are significant at 0.15 level for inclusion or exclusion. Logistic regression analyses were performed to determine the factors influencing fracture risk in the adolescents before and after adjusting for confounding variables. The maternal bone mass measurements used in the logistic regressions were converted to $\mathrm{Z}$-scores using the entire cohort of mothers as the reference group.

\section{Results}

Of the 3,273 neonates originally enrolled in the Bt20 cohort, fracture and bone mass data were available on 1,389 adolescents at age 17/18. Bone mass measurements were available on nearly all of their biological mothers $(\mathrm{WB}=1,383$ and $\mathrm{LS}=1,261$ ); however, information on previous fractures was only available on $688(\sim 50 \%)$ of these. There were no differences in age, anthropometric data and bone mass measurements between those mothers who did complete the fracture questionnaire and those who did not (data not shown). Figure 1 depicts the attrition of subjects in the cohort from birth until 17/18 years of age. The figure also shows the numbers of fracture questionnaires and bone mass measurements available at age $17 / 18$ on adolescent-biological mother pairs as well as the number of fracture questionnaires on the siblings of the 17/18-year-old adolescents.

Anthropometric and bone mass measurements

The baseline descriptive data of the adolescent-biological mother pairs of the different ethnic groups are shown in Tables 1 and 2. White adolescent males were heavier, had a greater BMI and were taller than black and MA adolescent males. White adolescent females were taller than black and MA adolescent females, but black adolescent females were heavier and had a greater BMI than the MA adolescent females.

After adjusting for height and weight, white males had a greater TB BA, LS BA and LS BMC than the males of the other ethnic groups. Mixed ancestry adolescent females had significantly lower TB BA than the black and white adolescent females. Adjusted TB BMC was not significantly different between the ethnic groups in either the adolescent males or females. Pubertal development was less advanced in black adolescent males than in other ethnic groups.

There were no differences in age or weight between the mothers in the different ethnic groups. White mothers were taller and had a lower BMI than their black and mixed ancestry peers. After adjusting for height and weight, black mothers had greater TB BA and BMC than mothers in the other two groups.

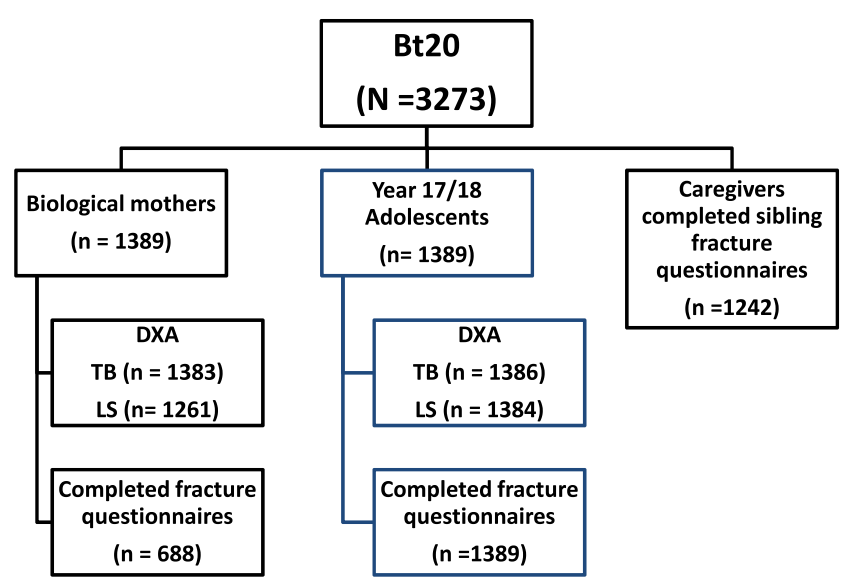

Fig. 1 Flow diagram describing the attrition of study participants from birth until 17/18 years of age including the number of adolescent-biological mother pairs and their siblings with fracture and bone mass data 
Table 1 Anthropometric and bone mass measurements of 17/18-year-old adolescents

\begin{tabular}{|c|c|c|c|c|c|c|c|c|c|c|c|c|c|c|}
\hline \multirow{3}{*}{$\begin{array}{l}\text { Anthropometric and bone mass } \\
\text { measurements }\end{array}$} & \multicolumn{4}{|c|}{ Whites } & \multicolumn{4}{|c|}{ Blacks } & \multicolumn{4}{|c|}{ Mixed ancestry } & \multicolumn{2}{|l|}{$p$ Values } \\
\hline & \multicolumn{2}{|c|}{ Males } & \multicolumn{2}{|c|}{ Females } & \multicolumn{2}{|c|}{ Males } & \multicolumn{2}{|c|}{ Females } & \multicolumn{2}{|c|}{ Males } & \multicolumn{2}{|c|}{ Females } & \multirow[t]{2}{*}{ Males } & \multirow[t]{2}{*}{ Females } \\
\hline & $n$ & $\begin{array}{l}\text { Mean } \\
(\mathrm{SD})\end{array}$ & $n$ & $\begin{array}{l}\text { Mean } \\
\text { (SD) }\end{array}$ & $n$ & $\begin{array}{l}\text { Mean } \\
\text { (SD) }\end{array}$ & $n$ & $\begin{array}{l}\text { Mean } \\
\text { (SD) }\end{array}$ & $n$ & $\begin{array}{l}\text { Mean } \\
(\mathrm{SD})\end{array}$ & $n$ & $\begin{array}{l}\text { Mean } \\
\text { (SD) }\end{array}$ & & \\
\hline Age (years) & 41 & $\begin{array}{l}17.8 \\
(0.3)\end{array}$ & 50 & $\begin{array}{l}17.8 \\
(0.2)\end{array}$ & 577 & $\begin{array}{l}17.9 \\
(0.4)\end{array}$ & 593 & $\begin{array}{l}17.9 \\
(0.4)\end{array}$ & 61 & $\begin{array}{l}18.2 \\
(0.5)\end{array}$ & 67 & $\begin{array}{l}18.2 \\
(0.5)\end{array}$ & $\begin{array}{l}\mathrm{MA}>\mathrm{B}^{*} \\
\mathrm{MA}>\mathrm{W}^{*}\end{array}$ & $\begin{array}{l}\mathrm{MA}>\mathrm{B}^{*} \\
\mathrm{MA}>\mathrm{W}^{*}\end{array}$ \\
\hline Weight (kg) & 41 & $\begin{array}{c}72.3 \\
(12.4)\end{array}$ & 50 & $\begin{array}{c}61.7 \\
(12.9)\end{array}$ & 577 & $\begin{array}{l}59.1 \\
(8.9)\end{array}$ & 590 & $\begin{array}{c}59.2 \\
(11.9)\end{array}$ & 61 & $\begin{array}{c}59.4 \\
(12.6)\end{array}$ & 67 & $\begin{array}{c}53.8 \\
(11.7)\end{array}$ & $\begin{array}{c}\mathrm{W}>\mathrm{B}^{*} \\
\mathrm{~W}>\mathrm{MA}^{*}\end{array}$ & $\begin{array}{l}\mathrm{W}>\mathrm{MA}^{* *} \\
\mathrm{~B}>\mathrm{MA}^{* *}\end{array}$ \\
\hline Height (m) & 41 & $\begin{array}{c}1.78 \\
(0.09)\end{array}$ & 50 & $\begin{array}{c}1.66 \\
(0.07)\end{array}$ & 577 & $\begin{array}{c}1.71 \\
(0.07)\end{array}$ & 590 & $\begin{array}{c}1.60 \\
(0.06)\end{array}$ & 61 & $\begin{array}{c}1.71 \\
(0.07)\end{array}$ & 67 & $\begin{array}{c}1.60 \\
(0.06)\end{array}$ & $\begin{array}{c}\mathrm{W}>\mathrm{B}^{*} \\
\mathrm{~W}>\mathrm{MA}^{*}\end{array}$ & $\begin{array}{c}\mathrm{W}>\mathrm{B}^{*} \\
\mathrm{~W}>\mathrm{MA}^{*}\end{array}$ \\
\hline BMI $\left(\mathrm{kg} / \mathrm{m}^{2}\right)$ & 41 & $\begin{array}{l}22.6 \\
(3.1)\end{array}$ & 50 & $\begin{array}{l}22.4 \\
(4.1)\end{array}$ & 577 & $\begin{array}{l}20.1 \\
(2.6)\end{array}$ & 590 & $\begin{array}{l}23.2 \\
(4.5)\end{array}$ & 61 & $\begin{array}{l}20.3 \\
(3.8)\end{array}$ & 67 & $\begin{array}{l}21.1 \\
(4.2)\end{array}$ & $\begin{array}{c}\mathrm{W}>\mathrm{B}^{*} \\
\mathrm{~W}>\mathrm{MA}^{* * *}\end{array}$ & $\mathrm{~B}>\mathrm{MA}^{*}$ \\
\hline TB BA $\left(\mathrm{cm}^{2}\right)$ & 41 & $\begin{array}{l}2,336.2 \\
(225.3)\end{array}$ & 50 & $\begin{array}{l}2,010.7 \\
(176.8)\end{array}$ & 577 & $\begin{array}{c}2,086 \\
(180.2)\end{array}$ & 593 & $\begin{array}{c}1,883 \\
(165.1)\end{array}$ & 61 & $\begin{array}{c}2,045 \\
(205.3)\end{array}$ & 67 & $\begin{array}{c}1,781 \\
(157.6)\end{array}$ & $\begin{array}{c}\mathrm{W}>\mathrm{B}^{*} \\
\mathrm{~W}>\mathrm{MA}^{*}\end{array}$ & $\begin{array}{c}\mathrm{W}>\mathrm{B}^{*} \\
\mathrm{~W}>\mathrm{MA}^{*} \\
\mathrm{~B}>\mathrm{MA}^{*}\end{array}$ \\
\hline Adjusted TB BA $\left(\mathrm{cm}^{2}\right)^{\mathrm{a}}$ & 41 & $\begin{array}{c}2,087.8 \\
(13.6)\end{array}$ & 50 & $\begin{array}{c}2,026.8 \\
(11.9)\end{array}$ & 577 & $\begin{array}{c}2,051.4 \\
(3.8)\end{array}$ & 590 & $\begin{array}{c}2,008.2 \\
(4.4)\end{array}$ & 61 & $\begin{array}{c}2,013.4 \\
(10.8)\end{array}$ & 67 & $\begin{array}{c}1,956.9 \\
(10.6)\end{array}$ & $\begin{array}{c}\mathrm{W}>\mathrm{B} * * * \\
\mathrm{~W}>\mathrm{MA}^{*} \\
\mathrm{~B}>\mathrm{MA}^{* * *}\end{array}$ & $\begin{array}{l}\mathrm{W}>\mathrm{MA}^{*} \\
\mathrm{~B}>\mathrm{MA}^{*}\end{array}$ \\
\hline TB BMC (g) & 41 & $\begin{array}{l}2,694.8 \\
(446.5)\end{array}$ & 50 & $\begin{array}{l}2,144.5 \\
(282.8)\end{array}$ & 577 & $\begin{array}{l}2,308.9 \\
(344.2)\end{array}$ & 593 & $\begin{array}{l}2,034.2 \\
(282.9)\end{array}$ & 61 & $\begin{array}{l}2,310.0 \\
(388.1)\end{array}$ & 67 & $\begin{array}{l}1,894.5 \\
(268.2)\end{array}$ & $\begin{array}{c}\mathrm{W}>\mathrm{B}^{*} \\
\mathrm{~W}>\mathrm{MA}^{*}\end{array}$ & $\begin{array}{l}\mathrm{W}>\mathrm{MA}^{*} \\
\mathrm{~B}>\mathrm{MA}^{* *}\end{array}$ \\
\hline Adjusted TB BMC $(\mathrm{g})+$ & 41 & $\begin{array}{c}2,354.2 \\
(37.2)\end{array}$ & 50 & $\begin{array}{c}2,158.6 \\
(32.4)\end{array}$ & 577 & $\begin{array}{c}2,277.5 \\
(10.4)\end{array}$ & 590 & $\begin{array}{c}2,185.3 \\
(12.0)\end{array}$ & 61 & $\begin{array}{c}2,280.9 \\
(29.5)\end{array}$ & 67 & $\begin{array}{c}2,130.9 \\
(28.9)\end{array}$ & NS & NS \\
\hline LS BA $\left(\mathrm{cm}^{2}\right)$ & 41 & $\begin{array}{l}68.9 \\
(6.2)\end{array}$ & 50 & $\begin{array}{l}57.8 \\
(5.4)\end{array}$ & 575 & $\begin{array}{l}62.7 \\
(6.0)\end{array}$ & 593 & $\begin{array}{l}54.5 \\
(5.9)\end{array}$ & 61 & $\begin{array}{l}61.8 \\
(5.6)\end{array}$ & 67 & $\begin{array}{l}53.2 \\
(5.8)\end{array}$ & $\begin{array}{c}\mathrm{W}>\mathrm{B}^{*} \\
\mathrm{~W}>\mathrm{MA}^{*}\end{array}$ & $\begin{array}{c}\mathrm{W}>\mathrm{B} * * \\
\mathrm{~W}>\mathrm{MA}^{*}\end{array}$ \\
\hline Adjusted LS BA $\left(\mathrm{cm}^{2}\right)^{\mathrm{a}}$ & 41 & $\begin{array}{l}62.8 \\
(0.8)\end{array}$ & 50 & $\begin{array}{l}58.8 \\
(0.7)\end{array}$ & 575 & $\begin{array}{l}60.7 \\
(0.2)\end{array}$ & 590 & $\begin{array}{l}58.8 \\
(0.2)\end{array}$ & 61 & $\begin{array}{l}60.0 \\
(0.6)\end{array}$ & 67 & $\begin{array}{l}57.8 \\
(0.6)\end{array}$ & $\begin{array}{c}\mathrm{W}>\mathrm{B}^{* *} \\
\mathrm{~W}>\mathrm{MA}^{* *}\end{array}$ & NS \\
\hline LS BMC (g) & 41 & $\begin{array}{l}71.8 \\
(12.6)\end{array}$ & 50 & $\begin{array}{c}56.1 \\
(10.0)\end{array}$ & 575 & $\begin{array}{c}58.3 \\
(10.8)\end{array}$ & 593 & $\begin{array}{l}53.1 \\
(9.6)\end{array}$ & 61 & $\begin{array}{c}59.0 \\
(10.9)\end{array}$ & 67 & $\begin{array}{l}50.1 \\
(8.5)\end{array}$ & $\begin{array}{c}\mathrm{W}>\mathrm{B}^{*} \\
\mathrm{~W}>\mathrm{MA}^{*}\end{array}$ & $\mathrm{~W}>\mathrm{MA}^{* * *}$ \\
\hline Adjusted LS BMC (g) ${ }^{\mathrm{a}}$ & 41 & $\begin{array}{l}62.8 \\
(1.4)\end{array}$ & 50 & $\begin{array}{l}56.8 \\
(1.2)\end{array}$ & 575 & $\begin{array}{l}56.7 \\
(0.4)\end{array}$ & 590 & $\begin{array}{l}58.0 \\
(0.5)\end{array}$ & 61 & $\begin{array}{l}57.6 \\
(1.1)\end{array}$ & 67 & $\begin{array}{l}56.5 \\
(1.1)\end{array}$ & $\begin{array}{c}\mathrm{W}>\mathrm{B}^{*} \\
\mathrm{~W}>\mathrm{MA}^{* *}\end{array}$ & NS \\
\hline \multicolumn{15}{|l|}{ Pubertal status } \\
\hline Stage 1 & 0 & $0 \%$ & 0 & $0 \%$ & 0 & $0 \%$ & 0 & $0 \%$ & 0 & $0 \%$ & 0 & $0 \%$ & & \\
\hline Stage 2 & 0 & $0 \%$ & 0 & $0 \%$ & 2 & $0.4 \%$ & 0 & $0 \%$ & 0 & $0 \%$ & 0 & $0 \%$ & $\mathrm{~W}>\mathrm{B}^{*}$ & \\
\hline Stage 3 & 1 & $2.4 \%$ & 5 & $10 \%$ & 74 & $13.8 \%$ & 81 & $14.3 \%$ & 3 & $7 \%$ & 4 & $9.3 \%$ & $\mathrm{MA}>\mathrm{B} * *$ & NS \\
\hline Stage 4 & 14 & $34.2 \%$ & 22 & $45 \%$ & 319 & $59.5 \%$ & 275 & $48.7 \%$ & 20 & $46.5 \%$ & 18 & $41.9 \%$ & & \\
\hline Stage 5 & 26 & $64.4 \%$ & 22 & $45 \%$ & 141 & $26.3 \%$ & 209 & $40.0 \%$ & 20 & $46.5 \%$ & 21 & $48.8 \%$ & & \\
\hline
\end{tabular}

Data are presented as number $(n)$ and percentage $(\%)$ or means (SD). Data compared between groups using ANOVA for continuous data and chi-square or Fisher's exact for categorical data

$N S$ not significant, $T B$ total body, $L S$ lumbar spine, $B A$ bone area, $B M C$ bone mineral content

$P$ values presented for ethnicity in male and females separately ( $W$ white, $B$ black, $M A$ mixed ancestry): ${ }^{*} p<0.001,{ }^{* *} \mathrm{p}<0.01,{ }^{* * *} p<0.05$

${ }^{\text {a }}$ Adjusted BA or BMC is adjusted for weight and height, and is presented as means

Predictors of BA and BMC in 17-18-year-old adolescents

To determine factors that made a significant contribution to adolescent TB and LS BA and BMC, ethnicity, gender, adolescent height, adolescent weight, Tanner stage (sub-divided into early or late puberty), maternal height, maternal weight, maternal TB and LS BA and BMC were chosen as candidate explanatory variables for the multivariate stepwise regression analyses. The results from regression models are presented in Table 3. Puberty was excluded from the analyses due to a lack of correlation. Including adolescent height, weight and maternal BA (except of TB that contributed minimally) and BMC resulted in the highest partial $R^{2}$ values for the respective adolescent bone variables. Maternal height and weight were 
Table 2 Anthropometric and bone mass measurements of mothers

\begin{tabular}{|c|c|c|c|c|c|c|c|}
\hline \multirow[t]{2}{*}{ Anthropometric and bone mass measurements } & \multicolumn{2}{|c|}{ Whites } & \multicolumn{2}{|c|}{ Blacks } & \multicolumn{2}{|c|}{ Mixed ancestry } & \multirow[t]{2}{*}{$p$ Value } \\
\hline & $n$ & Mean (SD) & $n$ & Mean (SD) & $n$ & Mean (SD) & \\
\hline Age (years) & 91 & $39.9(5.1)$ & 1,170 & $40.0(7.0)$ & 128 & $41.1(6.7)$ & NS \\
\hline Weight $(\mathrm{kg})$ & 91 & $72.2(16.4)$ & 1,165 & $75.7(16.3)$ & 127 & $73.8(16.5)$ & NS \\
\hline Height (m) & 91 & $1.65(0.06)$ & 1,165 & $1.59(0.06)$ & 127 & $1.59(0.07)$ & $\mathrm{W}>\mathrm{B}^{*}, \mathrm{~W}>\mathrm{MA}^{*}$ \\
\hline BMI $\left(\mathrm{kg} / \mathrm{m}^{2}\right)$ & 91 & $26.5(6.2)$ & 1,165 & $30.1(6.2)$ & 127 & $29.0(6.4)$ & $\mathrm{W}<\mathrm{B}^{*}, \mathrm{~W}<\mathrm{MA}^{* *}$ \\
\hline TB BA $\left(\mathrm{cm}^{2}\right)$ & 91 & $2,016.5(149.5)$ & 1,170 & $1,953.5(154.8)$ & 128 & $1,903.9(171.7)$ & $\mathrm{W}>\mathrm{B}^{*}, \mathrm{~W}>\mathrm{MA}^{*}, \mathrm{~B}>\mathrm{MA}^{* *}$ \\
\hline Adjusted TB BA $\left(\mathrm{cm}^{2}\right)^{\mathrm{a}}$ & 91 & $1,955.5(8.1)$ & 1,165 & $1,986.4(2.4)$ & 127 & $1,933.7(6.8)$ & $\mathrm{B}>\mathrm{W}^{*}, \mathrm{~B}>\mathrm{MA}^{*}, \mathrm{~W}>\mathrm{MA}^{* * *}$ \\
\hline TB BMC (g) & 91 & $2,229.5(276.9)$ & 1,170 & 2,211 (315.6) & 128 & $2,139(336.7)$ & $\mathrm{B}>\mathrm{MA} * * *$ \\
\hline Adjusted TB BMC $(\mathrm{g})^{\mathrm{a}}$ & 91 & $2,149.2(24.7)$ & 1,165 & $2,252.4(7.4)$ & 127 & $2,181.5(20.6)$ & $\mathrm{B}>\mathrm{W}^{*}, \mathrm{~B}>\mathrm{MA}^{* *}$ \\
\hline $\operatorname{LS~BA}\left(\mathrm{cm}^{2}\right)$ & 91 & $60.6(5.4)$ & 1,067 & $55.4(5.8)$ & 107 & $55(5.5)$ & $\mathrm{W}>\mathrm{B}^{*}, \mathrm{~W}>\mathrm{MA}^{*}$ \\
\hline Adjusted LS BA $\left(\mathrm{cm}^{2}\right)^{\mathrm{a}}$ & 91 & $58.0(0.5)$ & 1,064 & $57.1(0.2)$ & 106 & $55.8(0.4)$ & $\mathrm{W}>\mathrm{MA}^{*}, \mathrm{~B}>\mathrm{MA}^{* * *}$ \\
\hline LS BMC (g) & 91 & $61.5(10.7)$ & 1,067 & $56(10.8)$ & 107 & $55.1(10.7)$ & $\mathrm{W}>\mathrm{B}^{*}, \mathrm{~W}>\mathrm{MA}^{*}$ \\
\hline Adjusted LS BMC $(\mathrm{g})^{\mathrm{a}}$ & 91 & $58.1(1.0)$ & 1,064 & $58.1(0.3)$ & 106 & $56.6(0.9)$ & NS \\
\hline
\end{tabular}

Data are presented as means (SD). Data compared between groups using ANOVA for continuous data

$P$ values presented for ethnicity ( $W$ white, $B$ black, $M A$ mixed ancestry): ${ }^{*} p<0.001,{ }^{* *} p<0.01,{ }^{* * *} p<0.05$

$N S$ not significant, $T B$ total body, $L S$ lumbar spine, $B A$ bone area, $B M C$ bone mineral content

${ }^{\mathrm{a}}$ Adjusted BA or BMC is adjusted for weight and height, and presented as means (SE)

negative predictors of adolescent $\mathrm{BA}$ and $\mathrm{BMC}$, but contributed minimally to the overall variance. White ethnicity was a positive predictor of TB BA and BMC and LS BMC, and male gender was a positive predictor of TB BA and BMC and LS BA.
Factors associated with fractures in 17/18-year-old adolescents

Of the 1,389 adolescents with fracture data, $91(6.6 \%)$ were $\mathrm{W}, 1,170(84.2 \%)$ were B and $128(9.2 \%)$ were MA. Twenty-

Table 3 Regression models describing the relationship between predictors and adolescent bone area and bone mineral content

\begin{tabular}{|c|c|c|c|c|c|c|c|c|c|c|c|c|}
\hline & \multicolumn{3}{|c|}{ TB BA $(n=1,269)$} & \multicolumn{3}{|c|}{ TB BMC $(n=1,269)$} & \multicolumn{3}{|c|}{ LS BA $(n=1,169)$} & \multicolumn{3}{|c|}{ LS BMC $(n=1,169)$} \\
\hline & $\begin{array}{l}\text { Parameter } \\
\text { estimate }\end{array}$ & SE & $\begin{array}{l}\text { Partial } \\
R^{2}\end{array}$ & $\begin{array}{l}\text { Parameter } \\
\text { estimate }\end{array}$ & SE & $\begin{array}{l}\text { Partial } \\
R^{2}\end{array}$ & $\begin{array}{l}\text { Parameter } \\
\text { estimate }\end{array}$ & SE & $\begin{array}{l}\text { Partial } \\
R^{2}\end{array}$ & $\begin{array}{l}\text { Parameter } \\
\text { estimate }\end{array}$ & SE & Partial $R^{2}$ \\
\hline Intercept & -525.3 & 77.3 & & -672.2 & 190.5 & & -27.1 & 3.9 & & -28.9 & 7.4 & \\
\hline Whites & 39.21 & 9.6 & $0.002 *$ & 62.4 & 24.9 & $0.002 * *$ & - & & & 2.2 & 1.0 & $0.003 * *$ \\
\hline Males & 53.9 & 6.7 & $0.006^{*}$ & 115.6 & 17.4 & $0.018^{*}$ & 2.3 & 0.4 & $0.019 *$ & - & & \\
\hline $\begin{array}{l}\text { Adolescent height } \\
\text { (m) }\end{array}$ & $1,345.9$ & 42.5 & $0.660^{*}$ & $1,486.5$ & 110.3 & $0.409^{*}$ & 51.7 & 2.3 & $0.580^{*}$ & 47.8 & 3.0 & $0.275^{*}$ \\
\hline $\begin{array}{l}\text { Adolescent weight } \\
(\mathrm{kg})\end{array}$ & 8.47 & 0.2 & $0.170^{*}$ & 14.0 & 0.6 & $0.170^{*}$ & - & & & 0.25 & 0.02 & $0.051 *$ \\
\hline Late Tanner stage & - & & & 27.3 & 17.9 & 0.001 & - & & & - & & \\
\hline Maternal height (m) & -485.8 & 66.9 & $0.005^{*}$ & -709.4 & 132.4 & $0.007 *$ & -10.7 & 3.0 & $0.004 *$ & -14.1 & 5.0 & $0.003 * *$ \\
\hline Maternal weight (kg) & -1.4 & 0.2 & $0.003 *$ & -2.9 & 0.4 & $0.012 *$ & - & & & -0.03 & 0.02 & $0.004 * * *$ \\
\hline $\begin{array}{l}\text { Maternal bone } \\
\text { measurement }\end{array}$ & 0.32 & 0.03 & $0.004 *$ & 0.37 & 0.03 & $0.029 *$ & 0.29 & 0.03 & $0.021 *$ & 0.28 & 0.03 & $0.084 *$ \\
\hline Total $R^{2}$ & $0.852 *$ & & & $0.648^{*}$ & & & $0.624 *$ & & & $0.420^{*}$ & & \\
\hline
\end{tabular}

Mother's bone measurement corresponds to the respective TB or LS BA or BMC value for each column. All variables left in the model are significant at the 0.15 level

$T B$ total body, $B A$ bone area, $B M C$ bone mineral content, $L S$ lumbar spine

${ }^{*} p<0.001, * * p<0.05, * * * p<0.01$ 
two percent of the adolescents reported a history of having fractured a bone previously. The percentage of white children who reported fractures was double that of the other groups (W $42 \%$ vs. B $20 \%$ and MA $20 \%$; both $p<0.001$ ).

Twenty-two percent of adolescents who had siblings had a history of fractures. Of these adolescents who had fractures, $23 \%$ of their siblings had also sustained a fracture, while for those adolescents without a fracture history, only $14 \%$ of their siblings had fractures ( 23 vs. $14 \% ; p<0.01$ ).

Of the 688 biological mothers, who completed the fracture questionnaire, $60(9 \%)$ indicated that they had sustained a fracture before the age of 18 years (white mothers $31 \%$, mixed ancestry $16 \%$, black mothers $6 \%$; $\mathrm{W}>\mathrm{B}, p<0.001$; $\mathrm{MA}>\mathrm{B}, p=0.01)$. Unlike the pattern of fracture incidence among the adolescents and their siblings, there was no difference in the prevalence of fractures among the adolescents of mothers who had or did not have a history of fractures.

Bivariate logistic regression analyses were initially performed for the whole group to assess if any confounding variables, such as weight, height, ethnicity, gender, pubertal stage, adolescents' and mothers' BA and BMC (TB and LS), and sibling history of fracture or maternal history of fracture, were individually associated with adolescent fracture risk. In these analyses, the adolescent's risk of fracture was higher if a sibling had a history of fracture $(\mathrm{OR}=1.6,95 \% \mathrm{CI} 1.12-2.32$, $p=0.01$ ), but was not associated with maternal history of fracture $(\mathrm{OR}=1.09,95 \%$ CI $0.63-1.86, p=0.762)$. Neither adolescent weight nor pubertal stage was associated with fracture risk of the entire cohort; however, height was positively associated with the risk of fracture $(\mathrm{OR}=9.85,95 \% \mathrm{CI}$ 2.31-41.83, $p<0.01$ ), and males were at greater risk of fracture compared to females $(\mathrm{OR}=1.73,95 \%$ CI $1.33-2.24$, $p<0.001)$. Adolescent TB BA $(\mathrm{OR}=1.0008,95 \%$ CI $1.0002-1.001 ; p<0.05)$ and TB BMC $(\mathrm{OR}=1.0004,95 \% \mathrm{CI}$ $1.000002-1.0007, p<0.05$ ) were both marginally associated with increased fracture risk. Maternal LS BMC was inversely associated with fracture risk in their adolescent offspring $(\mathrm{OR}=0.80,95 \%$ CI $0.7-0.93 ; p<0.01)$. White adolescents had a greater risk of fracture than other ethnic groups $(\mathrm{OR}=2.82,95 \%$ CI $1.82-4.37, p<0.001)$.

Multivariate logistic regression analyses were performed on the entire group $(n=1099)$ to determine the risk factors for fractures in the adolescents. The factors which had been found to be significantly associated in simple logistic regression and multiple regression analyses were included in the model, namely gender, ethnicity, sibling history of fracture, adolescent and maternal heights, adolescent TB BA and BMC, and maternal LS BMC. Only the significant risk factors for adolescent fracture risk are shown in Table 4 . White ethnicity and male gender remained significant, with a greater risk of adolescent fracture. The adolescent's risk of fracture was $50 \%$ greater if a sibling had a history of fracture $(\mathrm{OR}=1.5,95 \% \mathrm{CI}$ $1.02-2.21, p<0.05)$. Maternal LS BMC was protective against
Table 4 Odds ratios for fractures in 17/18-year-old adolescents

\begin{tabular}{lll}
\hline Fractures $(n=1,099)$ & $\begin{array}{l}\text { Adjusted odds } \\
\text { ratio }\end{array}$ & $\begin{array}{l}95 \% \text { Confidence } \\
\text { interval }\end{array}$ \\
\hline Whites & $3.16^{*}$ & $1.89-5.32$ \\
Males & $1.94^{* *}$ & $1.25-2.99$ \\
Sibling history of fracture & $1.50^{* * *}$ & $1.02-2.21$ \\
Maternal LS BMC (Z-score) & $0.76^{* *}$ & $0.63-0.91$ \\
\hline
\end{tabular}

Odds ratios are adjusted for all other variables in the table and for adolescent-mother pair heights and adolescent TB BA and BMC

$L S$ lumbar spine, $B M C$ bone mineral content

${ }^{*} p<0.001,{ }^{* *} p<0.01, * * * p<0.05$

the risk of fracture in the adolescent $(24 \%$ reduction in fracture risk for every 1 unit increase in maternal BMC Zscore).

\section{Discussion}

To our knowledge, this is the first paper to describe the familial patterns of fracture risk in adolescents and its relationship with bone mass measurements in adolescent-biological mother pairs of different ethnic backgrounds. The main findings of this study were that an adolescent's risk of fracture was decreased if his/her mother had a greater lumbar spine BMC ( $24 \%$ reduction in fracture risk for every SD increase in maternal BMC), but was increased if a sibling had a history of fracture or if the adolescent was white or male. Adolescent height and weight, maternal BA and BMC, males and white ethnicity were positive predictors of adolescent bone mass. Lastly, there was a higher prevalence of fractures in white mothers prior to 18 years of age compared to the other ethnic groups, a pattern similar to that of their adolescent children, which we have reported previously [19]. However, we were unable to show any association between a maternal history of childhood/adolescent fractures and the prevalence of fractures in their adolescent offspring.

Maternal influences such as gestational height, adiposity and vitamin D status have been postulated to be important in intrauterine programming and in the tracking of skeletal development and body composition from infancy to adulthood $[20,21]$. These maternal influences are beyond the scope of this paper, but it will be important to determine if these factors predict or influence fracture risk and bone mass in adolescents from the different ethnic groups in South Africa.

Although the positive relationship between the mother's bone mass and her offspring's has been researched and documented worldwide [1, 22-24], the finding that maternal bone mass might influence her offspring's fracture prevalence during childhood and adolescence has not been reported 
previously. Intuitively, this association should not be surprising as several studies, although not all [25-28], have shown that children who had fracture(s) tend to have reduced BMC and BA compared to their peers who had no fractures, and genetic inheritance (maternal and paternal bone mass) plays a large role in determining childhood BMC, BA and peak bone mass [29]. However, in our earlier study of the Bt20 cohort [30], we did not find an inverse association between fracture history prevalence and bone mass at two time points during childhood and adolescence. In fact, in white males, there was a positive association between fracture risk and bone mass [30], possibly associated with increased contact sport participation [19]. Thus, the association between maternal LS BMC and adolescent fracture risk might be a proxy for structural differences in the adolescents, with low maternal BMC indicating poorer adolescent bone strength rather than differences in bone mass per se.

In addition to predicting adolescent fracture, maternal bone mass was also an independent predictor of adolescent BA and BMC. Twin- and family-based studies have indicated that 60 $85 \%$ of the variance in BMD is genetically determined [1, 22-24, 31]. All of these studies indicate that the bone mass of pre- and post-menarche daughters is related to the BMD of their mothers. Most workers have found correlations between 0.22 and 0.58 in parent/children pairs or mother/children pairs $[1,29]$. We found similar heritability rates (approximately $30 \%$ ) by maternal descent in pre-pubertal and early pubertal South African children [9], indicating that genetics plays an important role in determining bone mass in black, white and mixed ancestry South African children.

The pattern of differences in fracture prevalence between ethnic groups was similar in the biological mothers to that of their adolescent offspring, with the white mothers and adolescents reporting the highest prevalence of fractures (white mothers $31 \%$ vs. blacks $6 \%$ vs. MA $16 \%$ ). It is likely that the actual prevalence is higher than that recorded as the fractures were historic, occurred during childhood and had no means of verification. However, these figures are higher than those reported by an older group of men and women ( $>50$ years of age) participating in the European Prospective Osteoporosis Study (EPOS). They reported a fracture prevalence between the ages of 8 and 18 years of $8.9 \%$ in men and $4.5 \%$ in women [32]. We were unable to show any association between the history of childhood/adolescent fractures in mothers and the prevalence of fractures in their adolescent offspring within each ethnic group (data not shown). The findings support those of Ma and Jones who did not observe any association between the prevalence of childhood fractures in offspring and maternal fracture history (but the number of participants was small) [33]. However, we did show an association within the same family, as the prevalence of sibling fractures was significantly higher in families who had adolescents who had fractures $(23 \%)$ than in families whose adolescents had no fractures $(14 \%)$. Similar evidence of fracture association among siblings has been reported from Poland, where more than $50 \%$ of adolescents with multiple fractures indicated that at least one family member had sustained a fracture, while only $29 \%$ of the adolescents who had no fractures had a family member who had fracture(s) [34].

We were unable to show an association between the risk of childhood fractures and bone mass measurements at $17 /$ 18 years of age for the entire group. There are conflicting results concerning the association between childhood fractures and bone mass around the time of peak bone mass attainment. Several studies have found that childhood fractures are associated with low adult BMD [35],[36], but this was not confirmed by Kawalilak et al. [37]. The EPOS study conducted in over 50-year-old adults supports the latter study in that BMD was similar among those who did and did not report sustaining a fracture during childhood [32].

This study has several limitations. It relies heavily on the self-reporting of historical childhood fractures in adolescents, their siblings and their mothers. Being historical, we could not verify the occurrence of the fracture, its site, or if X-rays confirmed the presence of a fracture. Thus, we are dependent on memory of fracture events which is likely to be influenced by the severity of the fracture and the time between completing the questionnaire and the fracture event, which in the case of the mothers was at least 20 to 30 years. Potential differences in literacy between the black and white participants are not relevant as questionnaires were completed with the help of a research assistant. To assess data quality, the fractures were verified telephonically in $51(17 \%)$ of the adolescents who reported fractures. Forty-eight ( $94 \%$ ) confirmed having one or more fractures. Of the remaining three, two had reported strains as fractures, and one had reported no history of fractures in the initial questionnaire. Of the reported fractures, 46 $(96 \%)$ were said to have been diagnosed by a doctor, and one by a nursing sister. Eighty-nine percent (42/48) had confirmed that they had had a radiograph performed, three did not and two could not remember. Finally, this study did not include confounding variables such as vitamin D levels, calcium intake, physical activity scores or socioeconomic status, but the relationship between sports activities and fractures has been reported previously in this cohort [30].

\section{Conclusions}

We have shown that fracture history in South African adolescents is significantly associated with maternal bone mass as well as a fracture history in their siblings. There is also a strong ethnic component in fracture patterns within South Africa as the prevalence of fractures is higher in white South African families compared to the other ethnic groups. It has been reported that bone strength is lower in whites or Caucasians compared to other ethnic groups $[10,11]$, probably increasing their risk of fracture. 
Thus, further studies, using different techniques such as pQCT, are required to tease out the underlying physiological mechanisms for the differences in fracture rates among children of different ethnic groups within South Africa.

Acknowledgments Birth to Twenty is funded by the Wellcome Trust (UK), Medical Research Council of South Africa, Human Sciences Research Council of South Africa, National Research Foundation and the University of the Witwatersrand, Johannesburg. We are grateful to all the participants and their families in this study, and the entire Birth to Twenty team which includes interviewers, technicians, clerical workers, research scientists, nurses and receptionists.

\section{Conflicts of interest None.}

Open Access This article is distributed under the terms of the Creative Commons Attribution Noncommercial License which permits any noncommercial use, distribution, and reproduction in any medium, provided the original author(s) and the source are credited.

\section{References}

1. Krall EA, Dawson-Hughes B (1993) Heritable and life-style determinants of bone mineral density. J Bone Miner Res 8:1-9

2. Runyan SM, Stadler DD, Bainbridge CN et al (2003) Familial resemblance of bone mineralization, calcium intake, and physical activity in early-adolescent daughters, their mothers, and maternal grandmothers. J Am Diet Assoc 103:1320-1325

3. Ondrak KS, Morgan DW (2007) Physical activity, calcium intake and bone health in children and adolescents. Sports Med 37:587-600

4. Dotsch J (2011) Low birth weight, bone metabolism and fracture risk. Dermatoendocrinol 3:240-242

5. Javaid MK, Eriksson JG, Kajantie E et al (2011) Growth in childhood predicts hip fracture risk in later life. Osteoporos Int 22:69-73

6. Baird J, Kurshid MA, Kim M et al (2011) Does birthweight predict bone mass in adulthood? A systematic review and meta-analysis. Osteoporos Int 22:1323-34

7. Cooper C, Cawley M, Bhalla A et al (1995) Childhood growth, physical activity, and peak bone mass in women. J Bone Miner Res 10:940-947

8. Gafni RI, Baron J (2007) Childhood bone mass acquisition and peak bone mass may not be important determinants of bone mass in late adulthood. Pediatrics 119(Suppl 2):S131-6

9. Vidulich L, Norris SA, Cameron N et al (2011) Bone mass and bone size in pre- or early pubertal 10 -year-old black and white South African children and their parents. Calcif Tissue Int 88:281-93

10. Wetzsteon RJ, Hughes JM, Kaufman BC et al (2009) Ethnic differences in bone geometry and strength are apparent in childhood. Bone 44:970-975

11. Micklesfield LK, Norris SA, Pettifor JM (2011) Determinants of bone size and strength in 13-year-old South African children: the influence of ethnicity, sex and pubertal maturation. Bone 48:777-85

12. Baron JA, Barrett J, Malenka D et al (1994) Racial differences in fracture risk. Epidemiology 5:42-47

13. Barrett-Connor E, Siris ES, Wehren LE et al (2005) Osteoporosis and fracture risk in women of different ethnic groups. J Bone Miner Res 20:185-94

14. Solomon L (1968) Osteoporosis and fracture of the femoral neck in the South African Bantu. J Bone Joint Surg Br 50:2-13

15. Lei SF, Chen Y, Xiong DH et al (2006) Ethnic difference in osteoporosis-related phenotypes and its potential underlying genetic determination. J Musculoskelet Neuronal Interact 6:36-46
16. Richter L, Norris S, Pettifor J et al (2007) Cohort profile: Mandela's children: the 1990 Birth to Twenty study in South Africa. Int J Epidemiol 36:504-11

17. Tanner JM (1962) Growth at adolescence. Blackwell, Oxford

18. Norris SA, Richter LM (2005) Usefulness and reliability of Tanner pubertal self-rating to urban black adolescents in South Africa. J Res Adolesc 15:609-24

19. Thandrayen K, Norris SA, Pettifor JM (2009) Fracture rates in urban South African children of different ethnic origins: the Birth to Twenty cohort. Osteoporos Int 20:47-52

20. Ioannou C, Javaid MK, Mahon P et al (2012) The effect of maternal vitamin D concentration on fetal bone. J Clin Endocrinol Metab 97:E2070-E2077

21. Gale CR, Javaid MK, Robinson SM et al (2007) Maternal size in pregnancy and body composition in children. J Clin Endocrinol Metab 92:3904-11

22. Ferrari S, Rizzoli R, Slosman D et al (1998) Familial resemblance for bone mineral mass is expressed before puberty. J Clin Endocrinol Metab 83:358-61

23. Kuroda T, Onoe Y, Miyabara Y et al (2009) Influence of maternal genetic and lifestyle factors on bone mineral density in adolescent daughters: a cohort study in 387 Japanese daughter-mother pairs. J Bone Miner Metab 27:379-85

24. Ohta H, Kuroda T, Onoe Y et al (2010) Familial correlation of bone mineral density, birth data and lifestyle factors among adolescent daughters, mothers and grandmothers. J Bone Miner Metab 28:690-695

25. Clark EM, Tobias JH, Ness AR (2006) Association between bone density and fractures in children: a systematic review and metaanalysis. Pediatrics 117:e291-e297

26. Goulding A, Cannan R, Williams SM et al (1998) Bone mineral density in girls with forearm fractures. J Bone Miner Res 13:143-48

27. Goulding A, Jones IE, Taylor RW et al (2001) Bone mineral density and body composition in boys with distal forearm fractures: a dualenergy X-ray absorptiometry study. J Pediatr 139:509-15

28. Ma D, Jones G (2003) The association between bone mineral density, metacarpal morphometry, and upper limb fractures in children: a population-based case-control study. J Clin Endocrinol Metab 88:1486-91

29. Jouanny P, Guillemin F, Kuntz C et al (1995) Environmental and genetic factors affecting bone mass. Similarity of bone density among members of healthy families. Arthritis Rheum 38:61-67

30. Thandrayen K, Norris SA, Micklesfield LK et al (2011) Heterogeneity of fracture pathogenesis in urban South African children: the Birth to Twenty cohort. J Bone Miner Res 26:2834-42

31. Gueguen R, Jouanny P, Guillemin F et al (1995) Segregation analysis and variance components analysis of bone mineral density in healthy families. J Bone Miner Res 10:2017-22

32. Pye SR, Tobias J, Silman AJ et al (2009) Childhood fractures do not predict future fractures: results from the European Prospective Osteoporosis Study. J Bone Miner Res 24:1314-18

33. Ma DQ, Jones G (2002) Clinical risk factors but not bone density are associated with prevalent fractures in prepubertal children. J Paediatr Child Health 38:497-500

34. Konstantynowicz J, Bialokoz-Kalinowska I, Motkowski R et al (2005) The characteristics of fractures in Polish adolescents aged 16-20 years. Osteoporos Int 16:1397-403

35. Buttazzoni C, Rosengren EB, Tveit M et al (2013) Does a childhood fracture predict low bone mass in young adulthood? A 27-year prospective controlled study. J Bone Miner Res 28:351-59

36. Cheng S, Xu L, Nicholson PH et al (2009) Low volumetric BMD is linked to upper-limb fracture in pubertal girls and persists into adulthood: a seven-year cohort study. Bone 45:480-486

37. Kawalilak CE, Baxter-Jones AD, Faulkner RA et al (2010) Does childhood and adolescence fracture influence bone mineral content in young adulthood? Appl Physiol Nutr Metab 35:235-43 